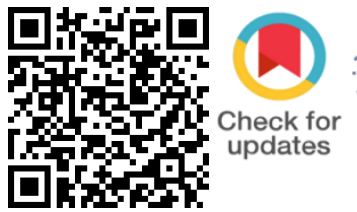

\title{
Accident Detection Using Mask R-CNN
}

\author{
Akshit Diwan ${ }^{1}$ | Vandit Gupta ${ }^{1}$ | Chaitanya Chadha ${ }^{2}$ \\ ${ }^{1}$ B-tech scholar, Department of CSE Maharaja Agrasen Institute of Technology, New Delhi, India \\ ${ }^{2} \mathrm{~B}$-tech scholar, Department of CSE SRM University, Haryana, India
}

\section{To Cite this Article}

Akshit Diwan, Vandit Gupta and Chaitanya Chadha, "Accident Detection Using Mask R-CNN", International Journal for Modern Trends in Science and Technology, Vol. 07, Issue 01, January 2021, pp.- 69-72.

\section{Article Info}

Received on 22-November-2020, Revised on 18-December-2020, Accepted on 28-December-2020, Published on 03-January-2021.

\section{ABSTRACT}

Deep learning is an artificial intelligence function that imitates the workings of the human brain in processing data and creating patterns for use in decision making. Deep learning is a subset of machine learning in artificial intelligence (AI) that has networks capable of learning and recognizing patterns from data that is unstructured or unlabeled. It is also known as deep neural learning or deep neural network. Convolutional Neural Networks (ConvNets or CNNs) are a category of Neural Networks that have proven very effective in areas such as image recognition and classification. ConvNets have been successful in identifying faces, objects and traffic signs apart from powering vision in robots and self-driving cars.

Consistently around the globe, an enormous number of individuals pass on from vehicle crash wounds. A large portion of the drivers are very much aware of the overall principles and security measures while driving yet it is just the laxity on their part, which causes mishaps and accidents. This paper helps in the detection of road accidents using the Mask R-CNN approach.

KEYWORDS: Deep Learning, Convolutional Neural Networks, Accident Detection

\section{INTRODUCTION}

Road traffic keeps on being a significant formative issue, a general well-being concern and is the main source of death and injury across the World killing more than 1.35 million internationally as revealed in the Global Status report on Road Safety 2018 with $90 \%$ of these setbacks occurring in the non-industrial nations.

The quickly developing populace, the remarkable pace of mechanization combined with the always developing urbanization has made individuals powerless against incessant road mishaps bringing about fatalities, wounds/handicaps. These mishaps in India kill practically 1.5 lakh individuals every year. Appropriately, India represents practically $11 \%$ of the accident-related deaths in the World. A lot of research has been conducted in this field over the past few years.

\section{RELATED WORK}

In 2012, Dong Yin [1] presented a method for the recognition of traffic accidents in real-time video images. In this paper, vehicle tracking was accomplished by matching the distance, area, and histogram of the same vehicle in the next frame. Using the concept of collision area and key point as pre-qualification, vehicle collision is detected. Huang He [2] presented a method for recognizing vehicles' collision by voice and designed a DSP-based vehicle collision warning device. SU Ruo-yu [3] focused on the reconstruction of vehicle's 
track, collision detection of vehicle, multi-angle observation of traffic accident on Vega platform in detail. Aköz Omer [4] presented a novel approach to describe traffic accident events at intersections in a human-understandable way using automated video processing techniques. Li Qiaoru [5] proposed a new prediction model of traffic accident black spots based on GA-BP neural network algorithm and rough set theory. To evaluate traffic accidents quantitatively, Hideki Fujii [6] had developed a cognitive error model and implemented it into a multi-agent-based traffic simulator. And more, Jules White [7] described how smartphones, could automatically detect traffic accidents using accelerometers and acoustic data immediately notified a central emergency dispatch server after an accident and provided situational awareness through photographs, GPS coordinates, VOIP communication channels, and accident data recording.

Based on the accumulation of our study works in this area for many years, this paper also tries to do some work in accident detection.

\section{METHODOLOGY}

The fundamental point of this paper is to help detect road accidents. The techniques used in this research paper are:

- Transfer Learning

- $\quad$ Mask R-CNN

\section{TRANSFER LEARNING}

Transfer learning is a machine learning technique where a model trained on one task is re-purposed on a second related task. This technique is an optimization that allows rapid progress or improved performance when modeling the second task. Transfer Learning is implemented by using a pre-trained model instead of training one from scratch. The weights of the model are extracted so that time is saved via training another model.

\section{OBJECT DETECTION}

Object Detection is the task of distinguishing cases of objects of a specific class inside a picture. This technique can be arranged into two fundamental sorts:

1) One-stage techniques

2) Two-stage techniques
One-stage techniques focus on inference speed. Some of the examples are YOLO, SSD, and RetinaNet. Two-stage techniques focus on detection accuracy. Some of the examples are Faster R-CNN, Mask R-CNN, and Cascade R-CNN.

\section{IMAGE SEGMENTATION}

Image segmentation is the process of partitioning an image into multiple segments (sets of pixels) which is typically used to locate objects and boundaries (lines, curves, etc.) in images. The result of this process is a set of segments that collectively cover the entire image or a set of contours extracted from the image which helps in edge detection.

Specifically Semantic Segmentation is used in Mask R-CNN for higher accuracy. Semantic segmentation is useful in detecting and classifying the object in an image when there is more than one class in the image as is the case in this paper.

\section{FAST REGIONS WITH CNN (FAST R-CNN)}

In Fast R-CNN, the input image is fed to CNN to generate a convolutional feature map. From which the region of proposals is identified and warped into squares. Via the RoI pooling layer, they are reshaped into a fixed size and fed into a fully connected layer. From the RoI feature vector, softmax layer is used to predict the class of the proposed region and offset values for the bounding box.

\section{FASTER REGIONS WITH CNN (FASTER R-CNN)}

In Faster R-CNN, the selective search algorithm is eliminated as compared to Fast R-CNN. The image is fed to CNN which generates a convolutional feature map. Due to the elimination of the selective search algorithm, a separate network is used to predict the region proposals. The RoI pooling layer is used to reshape predicted region

proposals which are then used to classify the image within the proposed region and predict the offset values for the bounding boxes.

\section{MASK REGIONS WITH CNN (MASK R-CNN)}

Mask R-CNN is a deep neural network that focuses on instance segmentation. It is implemented in two stages. At first, proposals about the regions are generated based on the presence of an object in the input image. For the implementation of the first stage, the Region proposal network (RPN) is used. Anchors are utilized to bind features to their raw image location.

In the second stage, the prediction of object class 
takes place, the bounding box is refined and a mask in the pixel level of the object is generated. For the implementation of the second stage, a Binary mask classifier is used to generate a mask for the object class. Instead of anchors, RoIAlign is used to locate the relevant features of the feature map.

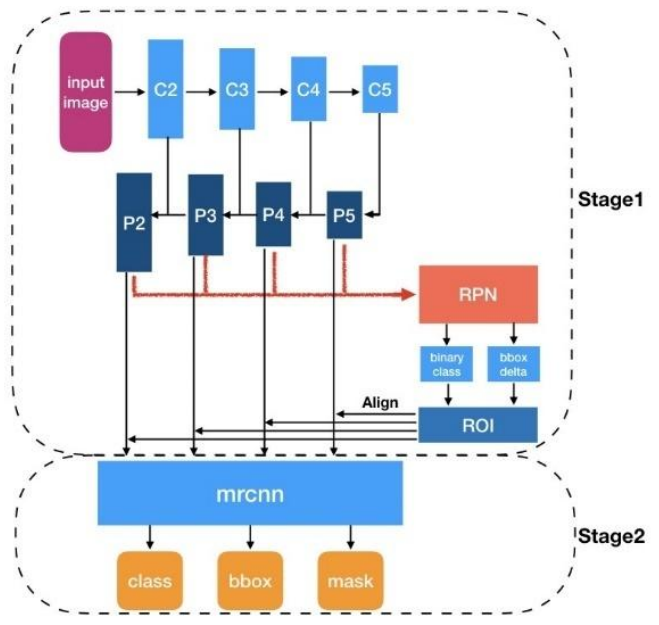

Figure 1: Mask R-CNN Architecture

In this paper, we are using Mask R-CNN for detecting vehicles. For the detection of a collision, we are using the concept of Intersection Over Union (IoU). It is calculated by finding the number of pixels where two objects overlap and dividing it by the number of pixels covered by both objects.

This gives us the measure of how much vehicle 1's bounding box is overlapping vehicle 2's bounding box. If the value

crosses a certain threshold (0.6, in our case) then a collision has occurred.

\section{DATASET USED}

$\mathrm{COCO}$ (Common Objects in Context) is large-scale object detection, segmentation, and captioning dataset. It contains over 12,000 images with cars already outlined. A sample image from the dataset can be observed in figure 2 below.

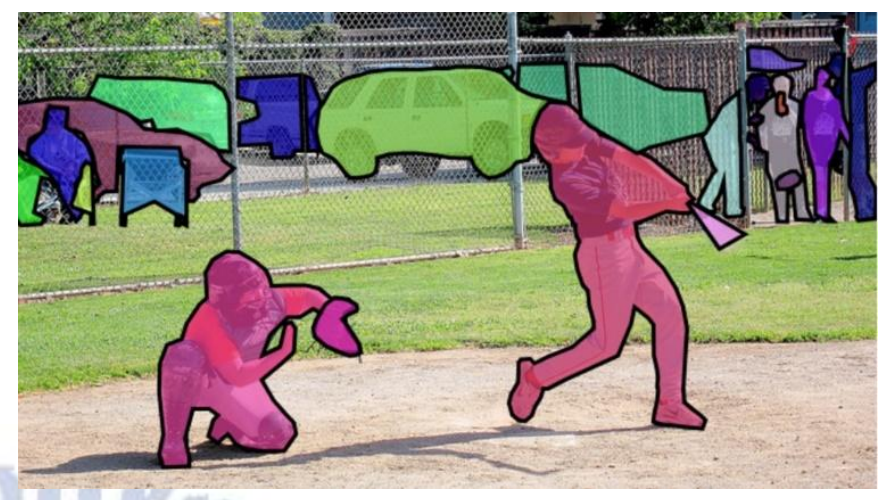

Figure 2: Sample image from COCO dataset

V. IMPLEMENTATION

Implementation of our approach is as follows:

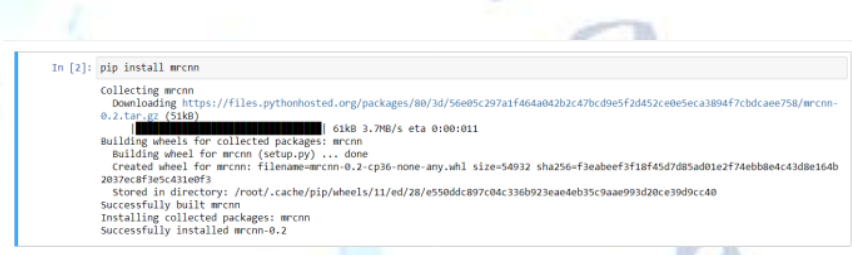

Figure 3: Installation of Mask R-CNN library

In figure 3, Mask R-CNN libraries are installed into the system using pip command.

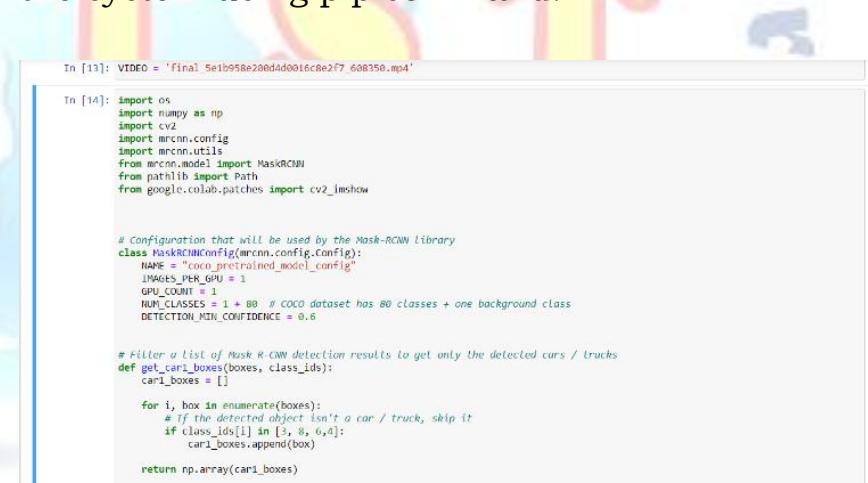

Figure 4: Configuration of Mask R-CNN for the filtration of vehicles

In figure 4, Mask R-CNN model is defined with certain parameters such as respective number of classes it has been trained on and minimum IOU confidence.

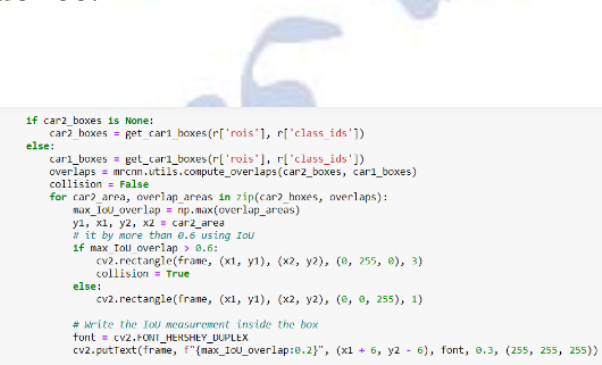

Figure 5: Computation of loU with threshold declaration for the collision

In figure 5 , the IoU calculations are implemented by taking overlaps for the two vehicles in contact with a threshold to check if there is a collision. 


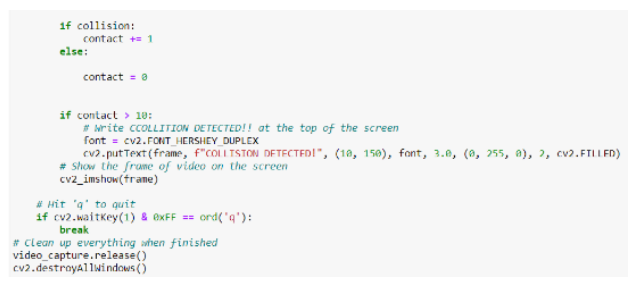

Figure 6: Detection of collision

In figure 6 , in case there is a hit, the model waits for 10 such continuous hits to ensure that this is an accident, and shows a message of collision detection.

\section{RESULTS}

The results of the implementation are as follows:

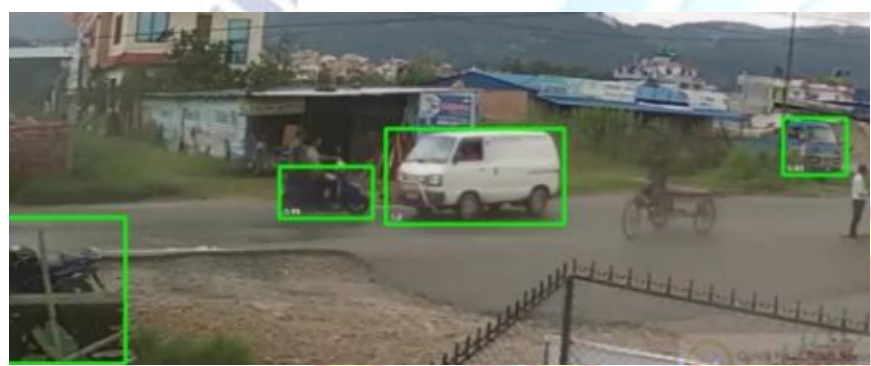

Figure 7: Before collision

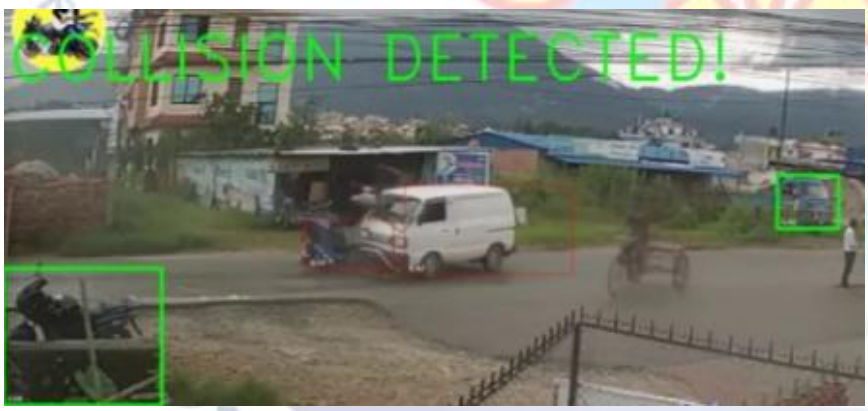

Figure 8: After collision

As can be seen from above images the color of the bounding boxes involved in collision changes from green to red as well when a collision has been detected.

\section{CONCLUSION}

It can be concluded that Mask R-CNN model is a viable model for the Accident Detection. It is producing great accuracy in detection and its Intersection over Union concept could be used to identify misshapen on the road and to help others which are around the accident spot. Combined with a well built Response system, this model would help avoid jams and save time and moreover, the faster the detection, faster the hospital nearby could be contacted as well as the family of the affected. This model is a reliable source for completion of the motive of this paper.

\section{REFERENCES}

[1] Dong Yin, Fan Zhang, Kun Wang (2012), "A Method for Traffic Collision Detection", Applied Mechanics and Materials Vols. 220-223 (2012) pp 2606-2610

[2] Huang He, Dai Shuo, Luo Hai, Zhou Heqin (2010), "A Collision Detection Device for Vehicle Based on Sound Signal”, Electronic Technology, V(08), pp: 65-67

[3] SU Ruo-yu, SUN Yong-rong, MIAO Jian-feng (2009), "Research and Realization of 3D Reconstruction System of Traffic Accident Based on Vega", Machinery \& Electronics, $\mathrm{V}(05)$, pp: 67-70

[4] Akoz Omer, M. Elif-Karsligil (2010), "Video-based traffic accident analysis at intersections using partial vehicle trajectories", International Conference on Image Processing, ICIP, p 4693-4696

[5] Li Qiaoru, Chen Liang, Cheng Changguang, Pan Yuexiang (2011), "A traffic accident predictive model based on neural networks algorithm and rough set theory", Applied Mechanics and Materials, v 97-98, p 947-951

[6] Fujii Hideki, Yoshimura Shinobu, Takano Yuya (2011), "Modeling of traffic accident in multi-agent-based traffic simulator", Transactions of the Japanese Society for Artificial Intelligence, v 26, n 1, p 42-49

[7] White Jules, Thompson Chris, Turner Hamilton, Dougherty Brian, Schmidt Douglas C. WreckWatch (2011), "Automatic traffic accident detection and notification with smartphones", Mobile Networks and Applications, v 16, n 3, p 285-303 